\section{Equivalência conceitual, de itens, semântica e operacional da versão brasileira do s-EMBU para aferição de práticas educativas parentais em adolescentes}

\author{
Conceptual, item, semantic, and operational \\ equivalence of a Brazilian version of the \\ $s$-EMBU for measuring parental rearing \\ practices in adolescents
}

Equivalencia conceptual, de ítems, semántica y operacional de la versión brasileña del s-EMBU para medir las prácticas educativas parentales en adolescentes

\begin{abstract}
Parental upbringing of children and adolescents has received little attention in research on the promotion of children's health and wellbeing. This paper presents the initial cross-cultural adaptation process of a Brazilian version of the $s$-EMBU for adolescents, which intends to capture parental rearing practices. The study takes a universalistic approach to assess the four initial phases of cross-cultural adaptation: conceptual, item, semantic, and operational equivalence. The process thus included a literature review, discussion with experts, and translation and back-translation of items. A pretest was conducted with 10 adolescents in the waiting room of a public clinic. The adolescents did not understand some items as proposed in the original constructs. After some rewording, the items were properly understood as projected by the original instrument. The findings suggest that this Brazilian version of $s-E M B U$ for adolescents is promising and that it provides room for future psychometric studies to complete the cross-cultural adaptation process.
\end{abstract}

Cross-Cultural Comparison; Child Rearing; Adolescent; Questionnaires
Paula Florence Sampaio 1 Claudia Leite Moraes 1 Michael Reichenheim 1

\section{Resumo}

O processo de educação de crianças e adolescentes por seus pais tem sido pouco valorizado em pesquisas que embasam ações de promoção de saúde e bem-estar infantil. Esta nota apresenta a primeira fase do processo de adaptação transcultural da versão brasileira para adolescentes do instrumento s-EMBU, que pretende aferir as práticas educativas parentais. Baseado no modelo universalista, este estudo focaliza as quatro primeiras etapas desse processo: avaliação das equivalências conceitual, de itens, semântica e operacional. Para tal, foram feitas revisão da literatura, discussão com especialistas, tradução $e$ retrotradução dos itens. Entretanto, pré-teste realizado com dez adolescentes revelou que alguns itens ainda não captavam os construtos subjacentes pretendidos. Após mudanças em sua redação, esses foram compreendidos corretamente, conforme proposto pelo instrumento original. Os achados apresentados indicam que essa versão brasileira do s-EMBU para adolescentes parece promissora, devendo ser realizados estudos psicométricos futuros a fim de complementar seu processo de adaptação transcultural.

Comparação Transcultural; Educação Infantil; Adolescente; Questionários 


\section{Introdução}

O processo de educação de crianças e adolescentes por seus pais envolve diferentes crenças e métodos, sendo influenciado por fatores inerentes aos pais, filhos e ao contexto em que vivem. Por meio das práticas educativas parentais, pais exercitam seus deveres parentais com objetivo de educar e socializar seus filhos 1. Diversos autores sugerem que práticas e estilo parental estão associados ao desenvolvimento psicológico e comportamental na infância, adolescência e idade adulta 2,3 .

$\mathrm{O}$ instrumento EMBU (Egna Minnen Beträffande Uppfostran - tradução livre de: Minhas Lembranças Quanto à Educação Infantil) é uma das propostas para aferição de práticas educativas parentais. Esse teve origem na Suécia e é composto por 81 itens, com quatro opções de resposta, respondidos pelo indivíduo adulto a respeito de suas memórias de criação parental na infância. Esses devem ser respondidos considerando pai e mãe separadamente ${ }^{4}$. A fim de facilitar seu uso em pesquisas envolvendo questionários multidimensionais, foram propostas diversas versões reduzidas do instrumento, também em outros idiomas, sendo a mais utilizada a de 23 itens, denominada s(short)-EMBU, desenvolvida por Arrindell et al. 5 . Avaliações psicométricas têm se mostrado robustas, corroborando três dimensões: rejeição, superproteção e calor emocional 5 .

Existem quatro versões em português do s-EMBU para adultos, sendo três brasileiras e uma portuguesa. No Brasil, encontra-se uso recente das versões de Zimmermann et al. 3 (adaptada de Gorayeb 6) e de Kobarg et al. 7 (adaptada da versão portuguesa 8). O estudo psicométrico de Kobarg et al. 7 demonstrou cargas fatoriais insuficientes para alguns itens, provavelmente devido à dissintonia na adaptação desses para o português 7 .

A versão "adulta" do EMBU foi adaptada para uso em adolescentes na Espanha a partir da mudança do tempo verbal de passado para presente 9 . Em 2012, um estudo psicométrico espanhol apontou que o s-EMBU poderia ser empregado em adolescentes 10. No Brasil, não há relatos de seu uso nessa faixa etária.

Apesar de relevantes no desenvolvimento de crianças e adolescentes, relações entre pais e filhos têm sido pouco valorizadas em pesquisas que objetivam embasar ações de promoção de saúde e bem-estar infantil. Visando estimular o crescimento do número de estudos sobre o tema, esta comunicação breve objetiva apresentar a primeira fase do processo de adaptação transcultural da versão brasileira para adolescentes do instrumento s-EMBU.

\section{Métodos}

A proposta de adaptação transcultural do s-EMBU para adolescentes aqui adotada adere ao modelo universalista de Herdman et al. 11 e Reichenheim \& Moraes 12 .

A avaliação das equivalências conceitual e de itens contemplou ampla revisão da literatura sobre conceitos que embasaram o instrumento original e sua aplicabilidade no contexto brasileiro. Posteriormente, discussão entre especialistas visou à avaliação da pertinência desses conceitos, bem como dos itens componentes no contexto adolescente.

A apreciação da equivalência semântica teve participação de tradutor nativo da língua inglesa, residente no Brasil, e de três pesquisadores brasileiros com inglês fluente, especialistas em Epidemiologia e Pediatria. No processo, a equipe usou as quatro versões disponíveis em português $3,6,7,8$ e a versão em inglês para adultos 5 . Optouse por selecionar a melhor tradução dentre as versões em português e, se necessário, redigir o item a partir da versão em inglês caso esse fosse julgado de difícil compreensão ou com problemas de equivalência semântica e/ou conceitual.

Uma vez consolidada, a versão síntese, consensuada entre pesquisadores e tradutor, foi retrotraduzida. Conforme sugerido na literatura, o tempo verbal dos itens foi modificado a fim de se aplicar o instrumento aos adolescentes e, consequentemente, aferir o relacionamento atual desses com seus pais.

Em seguida, foi realizado um pré-teste desse protótipo. Inicialmente, foi solicitado a dez adolescentes de 15 a 16 anos, que aguardavam atendimento no ambulatório geral de um hospital universitário, que preenchessem o instrumento em uma sala, separados de seus acompanhantes. Subsequentemente, perguntou-se sobre a facilidade de entendimento das instruções e itens de forma geral, e, a seguir, houve uma exploração quanto ao significado de cada item mediante pergunta aberta. Como reforço, solicitou-se também relato da situação abordada pelo item. Pretendeu-se, assim, avaliar se conceitos apreendidos pelos itens eram aqueles propostos no instrumento original, se a formulação desses permitia a correta compreensão pelos adolescentes e se as instruções de preenchimento estavam claras. Após alguns ajustes que se mostraram necessários, aplicou-se uma nova versão do instrumento a outros quatro adolescentes do mesmo cenário de atendimento, visando testar a adequação das alterações sugeridas. 


\section{Resultados}

À luz de extensa revisão da literatura e discussões de especialistas, entendeu-se que os conceitos relacionados ao processo de educação entre pais e filhos eram pertinentes ao contexto cultural brasileiro. Para seu uso em adolescentes, entretanto, fez-se necessário ajuste de alguns itens. Conforme mostra a Tabela 1, o item 6 necessitava de exemplos de atividades que fossem pertinentes aos adolescentes. As atividades "acampar" e "ir a clubes" não se aplicavam à boa parte dos adolescentes. Por isso, foram substituídas por “ir a lugares interessantes".
A fim de manter a equivalência semântica, alguns itens foram ligeiramente modificados em comparação a outras versões brasileiras para adultos. O item 5, por exemplo, deveria implicar na obrigação de se dar satisfação sobre o que se havia feito, a fim de captar controle excessivo exercido pelos pais. Por isso, incluiu-se a expressão "tinha que dar satisfação" com objetivo de harmonizar a tradução de "had to", que conota obrigação na língua inglesa. Já o item 13 tinha como tradução literal "eu era tratado como 'ovelha negra' ou 'bode expiatório”'. Porém, a fim de evitar estereótipos, a equipe optou por captar o significado dessas expressões usando os termos "o

Tabela 1

Versão em inglês para adultos, tradução consensual e versão final dos itens em português para população alvo adolescente do instrumento s-EMBU.

\begin{tabular}{|c|c|c|c|}
\hline Item & Versão em inglês para adultos & $\begin{array}{c}\text { Tradução consensual dos itens em português } \\
\text { para adolescentes }\end{array}$ & $\begin{array}{c}\text { Versão final dos itens em português para } \\
\text { adolescentes (após pré-teste) }\end{array}$ \\
\hline 1 & $\begin{array}{l}\text { It happened that my parents were sour or angry } \\
\text { with me without letting me know the cause. }\end{array}$ & $\begin{array}{l}\text { Meus pais ficam aborrecidos ou zangados } \\
\text { comigo sem me dizer os motivos. }\end{array}$ & $\begin{array}{l}\text { Meus pais ficam aborrecidos ou zangados } \\
\text { comigo sem me dizer os motivos. }\end{array}$ \\
\hline 2 & My parents praised me. & Meus pais me elogiam. & Meus pais me elogiam. \\
\hline 3 & $\begin{array}{l}\text { It happened that I wished my parents would } \\
\text { worry less about what I was doing. }\end{array}$ & $\begin{array}{l}\text { Eu gostaria que meus pais se preocupassem } \\
\text { menos com o que eu faço. }\end{array}$ & $\begin{array}{l}\text { Eu gostaria que meus pais se preocupassem } \\
\text { menos com o que eu faço. }\end{array}$ \\
\hline 4 & $\begin{array}{l}\text { It happened that my parents gave me more } \\
\text { corporal punishment than I deserved. }\end{array}$ & $\begin{array}{l}\text { Meus pais me dão mais castigos físicos } \\
\text { do que eu mereço. }\end{array}$ & $\begin{array}{l}\text { Meus pais me batem mais do que } \\
\text { eu mereço. }\end{array}$ \\
\hline 5 & $\begin{array}{c}\text { When I came home, I then had to account for } \\
\text { what I had been doing, to my parents. }\end{array}$ & $\begin{array}{l}\text { Quando eu chego em casa, tenho que dar } \\
\text { satisfação do que eu estava fazendo. }\end{array}$ & $\begin{array}{l}\text { Quando eu chego em casa, tenho que dar } \\
\text { satisfação do que eu estava fazendo. }\end{array}$ \\
\hline 6 & $\begin{array}{c}\text { I think that my parents tried to make my } \\
\text { adolescence stimulating, interesting and } \\
\text { instructive (for instance by giving me good } \\
\text { books, arranging for me to go on camps, taking } \\
\text { me to clubs). }\end{array}$ & $\begin{array}{l}\text { Meus pais tentavam tornar minha } \\
\text { adolescência estimulante, interessante, } \\
\text { instrutiva (por exemplo me dando livros pra } \\
\text { ler, me incentivando a conhecer/ir a lugares } \\
\text { interessantes, me levando com eles a passeios). }\end{array}$ & $\begin{array}{l}\text { Meus pais tentam tornar minha adolescência } \\
\text { estimulante, interessante, instrutiva (por } \\
\text { exemplo me dando livros pra ler, me } \\
\text { incentivando a conhecer/ir a lugares } \\
\text { interessantes/que eu gosto). }\end{array}$ \\
\hline 7 & $\begin{array}{l}\text { My parents criticized me and told me how lazy } \\
\text { and useless I was in front of others. }\end{array}$ & $\begin{array}{l}\text { Meus pais me criticam na frente dos outros, } \\
\text { dizendo que eu sou preguiçoso(a) e/ou inútil. }\end{array}$ & $\begin{array}{l}\text { Meus pais me criticam na frente dos outros, } \\
\text { dizendo que eu sou preguiçoso(a) e/ou inútil. }\end{array}$ \\
\hline 8 & $\begin{array}{c}\text { It happened that my parents forbade me to } \\
\text { do things other children were allowed to do } \\
\text { because they were afraid that something might } \\
\text { happen to me. }\end{array}$ & $\begin{array}{c}\text { Meus pais me proíbem de fazer coisas que } \\
\text { outros adolescentes podem fazer com } \\
\text { medo de que alguma coisa possa } \\
\text { acontecer comigo. }\end{array}$ & $\begin{array}{c}\text { Meus pais me proíbem de fazer coisas que } \\
\text { outros adolescentes podem fazer com } \\
\text { medo de que alguma coisa possa } \\
\text { acontecer comigo. }\end{array}$ \\
\hline 9 & $\begin{array}{l}\text { My parents tried to spur me to become } \\
\text { the best. }\end{array}$ & $\begin{array}{l}\text { Meus pais tentam me estimular para } \\
\text { que eu me torne o melhor. }\end{array}$ & $\begin{array}{l}\text { Meus pais me pressionam para que } \\
\text { eu me torne o melhor. }\end{array}$ \\
\hline 10 & $\begin{array}{c}\text { My parents would look sad or in some other } \\
\text { way show that I had behaved badly so that I got } \\
\text { real feelings of guilt. }\end{array}$ & $\begin{array}{l}\text { Meus pais parecem ficar tristes, ou de alguma } \\
\text { maneira mostram que eu me comporto mal, } \\
\text { fazendo com que eu me sinta culpado. }\end{array}$ & $\begin{array}{l}\text { Meus pais parecem ficar tristes, ou de alguma } \\
\text { maneira mostram que eu me comporto mal, } \\
\text { fazendo com que eu me sinta culpado. }\end{array}$ \\
\hline 11 & $\begin{array}{l}\text { I think that my parents' anxiety that something } \\
\text { might happen to me was exaggerated. }\end{array}$ & $\begin{array}{l}\text { Meus pais têm um medo exagerado de que } \\
\text { alguma coisa me aconteça. }\end{array}$ & $\begin{array}{l}\text { Meus pais têm um medo exagerado de que } \\
\text { alguma coisa me aconteça. }\end{array}$ \\
\hline 12 & $\begin{array}{l}\text { If things went badly for me, I then felt that my } \\
\text { parents tried to comfort and encourage me. }\end{array}$ & $\begin{array}{l}\text { Se as coisas vão mal para mim, eu sinto que } \\
\text { meus pais procuram me consolar } \\
\text { e me encorajar. }\end{array}$ & $\begin{array}{l}\text { Se as coisas vão mal para mim, eu sinto que } \\
\text { meus pais procuram me consolar } \\
\text { e me encorajar. }\end{array}$ \\
\hline 13 & $\begin{array}{l}\text { I was treated as the "black sheep" or } \\
\text { "scapegoat" of the family. }\end{array}$ & $\begin{array}{l}\text { Eu sou tratado(a) como "o(a) diferente" } \\
\text { ou "o(a) culpado(a) de tudo" da família. }\end{array}$ & $\begin{array}{l}\text { Eu sou tratado(a) como "o(a) diferente" } \\
\text { ou "o(a) culpado(a) de tudo" da família. }\end{array}$ \\
\hline
\end{tabular}

(continua) 
Tabela 1 (continuação)

\begin{tabular}{|c|c|c|c|}
\hline Item & Versão em inglês para adultos & $\begin{array}{c}\text { Tradução consensual dos itens em português } \\
\text { para adolescentes }\end{array}$ & $\begin{array}{c}\text { Versão final dos itens em português para } \\
\text { adolescentes (após pré-teste) }\end{array}$ \\
\hline 14 & $\begin{array}{c}\text { My parents showed with words and gestures } \\
\text { that they liked me. }\end{array}$ & $\begin{array}{l}\text { Meus pais demonstram com palavras e gestos } \\
\text { que gostam de mim. }\end{array}$ & $\begin{array}{l}\text { Meus pais demonstram com palavras e } \\
\text { gestos que gostam de mim. }\end{array}$ \\
\hline 15 & $\begin{array}{l}\text { I felt that my parents liked my brother(s) and/or } \\
\text { sister(s) more than they liked me. }\end{array}$ & $\begin{array}{l}\text { Eu sinto que meus pais gostam mais do(s) } \\
\text { meu(s) irmão(s) e/ou irmã(s) do que de mim. }\end{array}$ & $\begin{array}{l}\text { Eu sinto que meus pais gostam mais do(s) } \\
\text { meu(s) irmão(s) e/ou irmã(s) do que de mim. }\end{array}$ \\
\hline 16 & $\begin{array}{l}\text { My parents treated me in such a way that I felt } \\
\text { ashamed. }\end{array}$ & $\begin{array}{l}\text { Meus pais me tratam de um jeito que eu me } \\
\text { sinto envergonhado(a). }\end{array}$ & $\begin{array}{l}\text { Meus pais me tratam de um jeito que } \\
\text { eu sinto vergonha de mim mesmo(a). }\end{array}$ \\
\hline 17 & $\begin{array}{l}\text { I was allowed to go where I liked without my } \\
\text { parents caring too much. }\end{array}$ & $\begin{array}{c}\text { Eu posso ir onde eu quiser sem que meus } \\
\text { pais fiquem muito preocupados. }\end{array}$ & $\begin{array}{l}\text { Eu tenho permissão para ir onde eu quiser sem } \\
\text { que meus pais fiquem preocupados demais. }\end{array}$ \\
\hline 18 & $\begin{array}{l}\text { I felt that my parents interfered with everything } \\
\qquad \text { I did. }\end{array}$ & $\begin{array}{l}\text { Eu sinto que meus pais interferem em } \\
\text { tudo que eu faço. }\end{array}$ & $\begin{array}{c}\text { Eu sinto que meus pais interferem } \\
\text { em tudo que eu faço. }\end{array}$ \\
\hline 19 & $\begin{array}{l}\text { I felt that warmth and tenderness existed } \\
\text { between me and my parents. }\end{array}$ & $\begin{array}{l}\text { Eu sinto que existe carinho e afeto entre } \\
\text { mim e meus pais. }\end{array}$ & $\begin{array}{l}\text { Eu sinto que existe carinho e afeto entre } \\
\text { mim e meus pais. }\end{array}$ \\
\hline 20 & $\begin{array}{c}\text { My parents put decisive limits for what I was } \\
\text { and was not allowed to do, to which they then } \\
\text { adhered rigorously. }\end{array}$ & $\begin{array}{l}\text { Meus pais costumam colocar limites claros } \\
\text { sobre o que eu posso e o que eu não posso } \\
\text { fazer e seguem rigorosamente esses limites. }\end{array}$ & $\begin{array}{l}\text { Meus pais costumam colocar limites claros } \\
\text { sobre o que eu posso e o que eu não posso } \\
\text { fazer e seguem rigorosamente esses limites. }\end{array}$ \\
\hline 21 & $\begin{array}{c}\text { My parents would punish me hard, even for } \\
\text { trifles (small offenses). }\end{array}$ & $\begin{array}{l}\text { Meus pais me castigam com severidade, } \\
\text { mesmo por coisas sem importância. }\end{array}$ & $\begin{array}{l}\text { Meus pais me castigam com severidade, } \\
\text { mesmo por coisas sem importância. }\end{array}$ \\
\hline 22 & $\begin{array}{l}\text { My parents wanted to decide how I should be } \\
\text { dressed or how I should look. }\end{array}$ & $\begin{array}{c}\text { Meus pais querem decidir sobre como } \\
\text { devo me vestir ou sobre minha aparência. }\end{array}$ & $\begin{array}{l}\text { Meus pais querem decidir sobre como } \\
\text { devo me vestir ou sobre minha aparência. }\end{array}$ \\
\hline 23 & $\begin{array}{l}\text { I felt that my parents were proud when I } \\
\text { succeeded in something I had undertaken. }\end{array}$ & $\begin{array}{c}\text { Eu sinto que meus pais se orgulham quando eu } \\
\text { sou bem sucedido(a) em alguma coisa que } \\
\text { eu me proponho a fazer. }\end{array}$ & $\begin{array}{l}\text { Eu sinto que meus pais se orgulham quando } \\
\text { eu sou bem sucedido(a) em alguma coisa } \\
\text { que eu me proponho a fazer. }\end{array}$ \\
\hline
\end{tabular}

diferente" ou "o culpado de tudo". Alguns termos formais foram substituídos por outros de mais fácil compreensão para adolescentes, destacando-se "calor e ternura" por "carinho e afeto", e os verbos "desejaria" e "há" por "gostaria" e "existe", respectivamente.

Apesar desses cuidados, durante o pré-teste, identificou-se que os itens 9 e 16 não foram totalmente compreendidos pelos adolescentes. $\mathrm{O}$ item 9 foi o que mostrou maior dificuldade de adaptação, já que, no instrumento sueco, o verbo empregado é sporra, que tem conotação negativa naquele país. Dessa maneira, a tradução deveria indicar pressão ou cobrança excessiva feita pelos pais para que seu filho fosse o melhor. Só que, no pré-teste, ao contrário, observou-se que o verbo foi entendido como um estímulo realizado pelos pais. Por isso, optou-se pela tradução "meus pais me pressionam para que eu me torne o melhor", utilizada por Zimmermann et al. 3 .

Já o item 16 foi, inicialmente, traduzido como “...de um jeito que eu me sinto envergonhado(a)”, remetendo aos adolescentes uma ideia de vergonha dos pais perto de amigos, como quando são "tratados como bebês" na frente de seus colegas. Para que o item mantivesse uma equivalência conotativa à proposta original, se referindo à re- jeição por parte dos pais, optou-se por “...de um jeito que eu sinto vergonha de mim mesmo(a)".

O item 4 sobre punição corporal foi entendido pelos adolescentes especificamente como "apanhar dos pais", ainda que a pergunta original sugerisse castigos físicos em geral. Assim sendo, optou-se por uma adaptação mais direcionada: “...me batem mais do que eu mereço”. Já modificado em uma etapa anterior, o item 6 precisou ser aparado novamente após o pré-teste. Os adolescentes reportaram não ser estimulante ir passear com os pais e, por isso, a alternativa foi retirada. O item 17 também foi aprimorado ao se escolher o termo "tenho permissão" em vez de "eu posso", já que eles referiam que nunca poderiam sair sem a permissão dos pais.

Após esses vários ajustes, a versão "propositiva”, apresentada na terceira coluna da Tabela 1 , foi bem aceita pelos adolescentes. Ademais, assegurou-se equivalência operacional, uma vez que todos os adolescentes entenderam as instruções do instrumento. Essa era uma preocupação da equipe, já que a marcação de itens para pais e mães necessita ser em linhas distintas. 


\section{Conclusão}

O presente artigo reforça o valor das etapas de avaliação das equivalências conceitual, de itens, semântica e operacional, bem como da subsequente realização de pré-teste quando se pretende adaptar instrumento elaborado em outro contexto cultural. Essas etapas trazem a oportunidade de detecção de falhas no processo que, se não forem resolvidas, podem levar à má classificação de indivíduos e, possivelmente, a dificuldades de comparações externas. Os achados apresentados indicam que essa versão brasileira do s-EMBU para adolescentes parece promissora. Estudos psicométricos futuros que envolvam apreciação da confiabilidade, validade de estrutura dimensional e de construto via testes de hipótese deverão ser realizados visando complementar o processo de adaptação transcultural dessa nova versão do s-EMBU em português 12 .

\section{Resumen}

El proceso de educación de niños y adolescentes, llevado a cabo por los padres, se ha infravalorado en investigaciones que apoyan acciones para promover la salud y el bienestar infantil. Esta nota presenta la primera etapa del proceso de adaptación transcultural de la versión brasileña del instrumento s-EMBU para adolescentes, cuyo objetivo es evaluar las prácticas educativas parentales. Basado en el modelo universalista, este estudio se centra en la evaluación de las equivalencias conceptual, de ítems, semántica y operacional. Para este propósito, se hizo una revisión de literatura, debates con expertos, traducción y retrotraducción de ítems. Sin embargo, la pre-prueba realizada con 10 adolescentes reveló que algunos ítems no habían sido entendidos como proponen los constructos subyacentes. Después de cambios en la redacción, los ítems se entendieron según lo propuesto por el instrumento original. Los resultados presentados indican que esta versión brasileña de s-EMBU para adolescentes parece prometedora y se deben realizar futuros estudios psicométricos para complementar el proceso de adaptación transcultural.

Comparación Transcultural; Crianza del Niño;

Adolescente; Cuestionarios

\section{Colaboradores}

P. F. Sampaio colaborou na pesquisa bibliográfica, concepção e delineamento do estudo, coleta de dados, análise de dados, interpretação de resultados e redação do texto. C. L. Moraes e M. Reichenheim colaboraram na concepção e delineamento do estudo, análise de dados e interpretação de resultados e revisão crítica do texto. 


\section{Referências}

1. Darling N, Steinberg L. Parenting style as a context: an integrative model. Psychol Bull 1993; 113: 487-96.

2. Benchaya MC, Bisch NK, Moreira TC, Ferigolo M, Barros HM. Non-authoritative parents and impact on drug use: the perception of adolescent children. J Pediatr (Rio J.) 2011; 87:238-44.

3. Zimmermann JJ, Eisemann MR, Fleck MP. Is parental rearing an associated factor of quality of life in adulthood? Qual Life Res 2008; 17:249-55.

4. Perris C, Jacobsson L, Lindstrom H, von Knorring L, Perris H. Development of a new inventory assessing memories of parental rearing behaviour. Acta Psychiatr Scand 1980; 61:265-74.

5. Arrindell WA, Sanavio E, Aguilar G, Sica C, Hatzichristou C, Eisemann M, et al. The development of a short form of EMBU: its appraisal with students in Greece, Guatemala, Hungary and Italy. Pers Individ Dif 1999; 27:613-28.

6. Gorayeb R. Child rearing patterns in Brazil. Acta Psychiatr Scand Suppl 1988; 344:147-9.

7. Kobarg APR, Vieira V, Vieira ML. Validação da Escala de Lembranças Sobre Práticas Parentais (EMBU). Aval Psicol 2010; 9:77-85.
8. Canavarro MCS. A avaliação das práticas educativas através do EMBU: estudos psicométricos. Revista Psychologica 1996; 16:5-18.

9. Castro J, Toro J, Arrindell WA, van der Ende J, Puig J. Perceived parental rearing style in Spanish adolescents, children and parents: three new forms of the EMBU. In: Costa S, editor. Psychiatry: a world perspective. Amsterdam: Elsevier; 1990. p. 345-49.

10. Penelo E, Viladrich C, Domenech JM. Adolescents perceptions of parental behavior: psychometric properties of the short Egna Minnen Betraffande Uppfostran-Adolescent version (S-EMBU-A) in a clinical sample. Compr Psychiatry 2012; 53:87-94.

11. Herdman M, Fox-Rushby J, Badia X. A model of equivalence in the cultural adaptation of HRQoL instruments: the universalist approach. Qual Life Res 1998; 7:323-35.

12. Reichenheim ME, Moraes CL. Operacionalização de adaptação transcultural de instrumentos de aferição usados em epidemiologia. Rev Saúde Pública 2007; 41:665-73.

Recebido em 10/Mar/2014

Aprovado em 19/Mai/2014 